\title{
A classification of NOergic neurons in the inferior colliculus of rat according to co-existence with classical amino acid transmitters
}

\author{
By \\ Men-dar WU ${ }^{1}$, Masaru KIMURA ${ }^{2}$, \\ Ishigami HIROMICHI ${ }^{3}$ and Robert H. HELFERT ${ }^{4}$ \\ ${ }^{1}$ Research Complex for the Medicine Frontiers, ${ }^{2}$ Department of Anatomy \\ ${ }^{3}$ Department of Otorhinolaryngology, Aichi Medical University School of Medicine \\ ${ }^{4}$ Department of Surgery, Southern Illinois University School of Medicine
}

- Received for Publication, February 20, 2008 -

\begin{abstract}
Key Words: NO, GABA, Glutamate, inferior colliculus
\end{abstract}
\begin{abstract}
Summary: Since the localization of nitric oxide synthase (NOS) can be identified by enzyme histochemistry for NADPH-diaphorse (NADPH-d), this method has been used widely for mapping NOS-containing (presumably NOergic) neurons in the central nervous system. So far several studies suggest that NADPH-d is present in distinct neuronal populations in the inferior colliculus (IC), a major processing center for both the ascending and descending auditory pathway, and NO may play an important role in audition. On one hand, there is evidence from several lines of research that the IC makes extensive use of the neuroactive amino acids, in particular the inhibitory transmitter g-aminobutyric acid (GABA) and the excitatory amino acid glutamate (GLU). However, lacking is a description of the distribution of NOergic neurons to which traditional neurotransmitters may be linked. The present research utilized NADPH-d enzyme histochemistry in combination with immunocytochemistry to determine if NO may colocalize with either or both GABA and glutamate in distinct subpopulations of IC neurons.

The NADPH-d positive neurons were predominantly found in two main subdivisions of the IC: the external cortex (ECIC) and the dorsal cortex (DCIC). The large numbers of these NADPH-d positive neurons appeared immunostained for GLU while only a small number, seemed to belong to the small cells (somatic area $<100 \mu \mathrm{m}^{2}$ ) similarity to stellate cells group was positive for GABA throughout the cortex of the IC. Owing to no coexistence between GABA and GLU in the same NADPH-d positive neuron in the pairs of adjacent sections of the IC by the mirror-image technique, the present results consequently support that NOergic neurons could be subdivided into at least three distinct populations with a large proportion of about $77 \%$ being GLUergic, much lower frequency of about $11 \%$ being GABAergic and the remaining $12 \%$ expressing non-GABA and non-GLU.

In summary, the existence of two functionally distinct populations of NO/GABAergic and NO/GLUergic neurons in the NOergic neurons of IC suggest that at least two differential pattern of GLU-mediated excitatory NO transmission and GABA-mediated inhibitory NO transmission are involved in the networking of auditory communication in the cortex of IC.
\end{abstract}

\section{Introduction}

Since the first report suggesting its involvement in signal transduction in the brain ${ }^{15}$, nitric oxide (NO) has been established as a neuroactive substance with a biochemical mechanism of action radically different from that of traditional neurotransmitters. Nitric oxide is an inorganic, gaseous free radical that mediates a variety of functions, including vasodilatation, neurotransmission and cytotoxicity. The nitric oxide synthase (NOS) fam- ily catalyzes the oxidation of arginine to form 1-citrulline and NO. Each NOS isoform possesses regions for the binding of calmodulin and reduced nicotine-adenine-nucleotide phosphate (NADPH). NOS can be identified by histochemical staining for NADPH diaphorase (NADPHd) ${ }^{3,9}$. Indeed, the above approach has been used widely for mapping NOS-containing (presumably NOergic) neurons in the central nervous system ${ }^{3,38}$. NADPH-d histochemistry has also been combined with immunohistochemistry to assess relationships between the distribu-

Corresponding author: Men-dar Wu, MD, Research Complex for the Medicine Frontiers, Aichi Medical University School of Medicine, 21 Karimata, Yazako, Nagakute-Cho, Aichi, 480-1195 Japan, Email: mendwugo@aichi-med-u.ac.jp 
tion of NO and traditional neurotransmitters in various regions of the $\mathrm{CNS}^{1,14,30,35,37)}$.

Several studies suggest that NO and NOS play a role in audition. Nitric oxide synthase (not differentiated by isoform) was observed in cells and great numbers of synaptic boutons in nuclei of the lower auditory brainstem ${ }^{20}$ and inferior colliculus (IC) ${ }^{4)}$. In the cochlea, NOS expression has been described in the spiral ganglion and organ of Corti ${ }^{18)}$. In mice, immunostaining for NOS increases with age in the spiral ganglion ${ }^{40)}$, suggesting that there is an elevation in NO synthesis in the primary afferent auditory neurons of aged animals.

The IC is a major processing center for both the ascending and descending auditory pathway. In rats, glucose utilization measurements indicate that the IC is one the most metabolically active areas in the brain ${ }^{34)}$. Several studies suggest that NADPH-d is present in distinct neuronal populations in the $\mathrm{IC}^{8,38)}$. However, lacking is a description of the distribution of NOergic neurons to which traditional neurotransmitters may be linked.

Despite the fact that the functional role of some neurotransmitters and neuromodulators in the IC is still a matter of dispute, there is evidence from several lines of research that the IC makes extensive use of the neuroactive amino acids, in particular the inhibitory transmitter g-aminobutyric acid (GABA) and the excitatory amino acid glutamate $(\mathrm{GLU})^{2,10,19,22,23,26)}$. The present research utilized NADPH-d histochemistry in combination with immunocytochemistry to determine if NO may colocalize with either or both GABA and GLU in distinct subpopulations of IC neurons.

\section{Materials and Methods}

\section{Tissue preparation}

Thirteen 300-400 g, male Fischer-344 (F344) rats were used in this study. They were deeply anesthetized with a cocktail of ketamine $(80 \mathrm{mg} / \mathrm{kg}$ body weight $)$ and xylazine $(10 \mathrm{mg} / \mathrm{kg}$ body weight) administered intraperitoneally, exsanguinated transcardially with $\sim 50 \mathrm{ml}$ of physiological saline containing $0.01 \%$ sodium nitrite, and perfused with $\sim 750 \mathrm{ml}$ of aldehyde-based fixative in Sorensen's sodium-potassium phosphate buffer, pH 7.4. For the experiments in which only NADPH-d histochemistry was performed, three animals were perfused with a fixative containing $4 \% \mathrm{w} / \mathrm{v}$ freshly depolymerized paraformaldehyde (PAF). For studies combining NADPH-d histochemistry and immunocytochemistry, ten animals were perfused with fixative containing $4 \% \mathrm{w} / \mathrm{v}$ PAF and $0.05-0.1 \% \mathrm{v} / \mathrm{v}$ glutaraldehyde. Following perfusion, the brains were quickly removed and the midbrain dissected, immersed in the same fixative for an additional $2 \mathrm{hr}$, and stored overnight at $4^{\circ} \mathrm{C}$ in $0.005 \mathrm{M}$ phosphate-buffered saline (PBS, pH 7.4) containing 30\% w/v sucrose. Using a sledge microtome (AO Spencer), $6 \mu \mathrm{m}$ frozen sections were cut through the right and left ICs along sagittal and frontal planes, respectively, and collected in cold, 0.05 $\mathrm{M}$ Tris- $\mathrm{HCl}$ buffer ( $\mathrm{pH}$ 8.0). All of the selected mixedaldehyde-fixed sections were first reacted for NADPH-d, and then pairs of adjacent sections were respectively immunolabeled for GABA and GLU as described below.

\section{NADPH-d histochemistry}

The NADPH-d procedure was modified from SchererSingler et al ${ }^{33)}$ Briefly, after washing in $0.1 \mathrm{M}$ Tris- $\mathrm{HCl}$ buffer ( $\mathrm{pH}$ 8.0) free-floating sections were incubated in a solution containing $0.25 \mathrm{mg} / \mathrm{ml}$ nitroblue tetrazolium (NBT), $1 \mathrm{mg} / \mathrm{ml} \beta$-NADPH and $0.3 \%$ Triton $\mathrm{X}-100$ in $0.1 \mathrm{M}$ Tris- $\mathrm{HCl}$ buffer ( $\mathrm{pH} 8.0$ ) for $30-45 \mathrm{~min}$ at $37^{\circ} \mathrm{C}$ in the dark. The aforementioned chemicals were purchased from Sigma (St. Louis, MO). The reaction was terminated by transferring the sections through a series of graded acetones, which also proved useful in removing the residual formazan and reducing non-specific background signal. Finally, the sections were washed in PBS. Sections reacted with incubation media free of the substrate $(\beta-N A D P H)$ or NBT served as controls.

\section{Immunocytochemistry for GABA and GLU}

Following the NADPH-d reaction, alternate mixedaldehyde-fixed sections were preincubated for one hour in a blocking solution of $10 \%$ normal goat serum (NGS, Vector Laboratories, Burlingame, CA) in PBS. The sections were then gently agitated in an affinity-purified polyclonal rabbit antiserum against GABA conjugate (provided by Robert J. Wenthold, Ph.D., NIDCD, NIH, Bethesda, MD) ${ }^{41}$, diluted $1: 1000$ in PBS containing $5 \% \mathrm{NGS}$, for $12-18 \mathrm{hr}$ at $4{ }^{\circ} \mathrm{C}$. Adjacent sections were blocked for one hour in 10\% normal horse serum (NHS, Vector Laboratories) in PBS and then incubated in a monoclonal mouse antiserum against GLU (abcam ${ }^{\circledR}$, ab8893), diluted 1:1750 in PBS with 5\% NHS under the same conditions and time period as those described for GABA. The primary immunolabeling was tagged with biotinylated horseradish peroxidase using Vectastain ABC kits (Vector Laboratories), and the peroxidase activity was visualized using a solution of $0.05 \mathrm{M} \mathrm{3,3}$, -diaminobenzidine tetrahydrochloride (DAB) and 0.005\% hydrogen peroxide in PBS. Control sections were incubated with 5\% normal sera in PBS in the absence of primary antisera.

\section{Evaluation of labeled neurons}

After completion of the above procedures, the labeled sections were rinsed in PBS, mounted on gelatin-coated slides and allowed to air dry. On the following day, slides with tissue reacted for NADPH-d only were counterstained with neutral red. After the slides were coverslipped, the IC sections were examined and photographed using a Leitz Diaplan photomicroscope equipped with bright field optics (Leica, Wetzlar, Germany). With the 
same microscope, camera lucida images were traced from five respective pairs of sagittal sections obtained from a mediolateral extent of the IC corresponding roughly to that of "lat. $2.0 \mathrm{~mm}$ " in Faye-Lund and Osen ${ }^{11)}$. Each pair consisted of one section colabeled for NADPH-d and GABA, and the other for NADPH-d and GLU. Adjacent surfaces were examined as mirror-images to determine if any of the NADPH-d-positive coimmunolabeled for GABA and GLU. The camera lucida images were then optically scanned and digitized using a G4 Power Mac (Apple Computer, Inc., Cupertino, CA) running the public domain NIH Image program (developed at the U.S. National Institutes of Health and available on the Internet at). The distribution of labeled neurons was mapped, with each neuron categorized into one of three classes: NADPH-d+/0, NADPH-d+/GABA+, and NADPH+/GLU+. Somatic areas were measured from those NADPH-positive neurons possessing discernible nuclear profiles.

\section{Results}

The NADPH-d staining pattern observed in the IC of rat was consistent with that observed in a study by Druga and $\mathrm{Syka}^{8)}$, in which tissues were fixed with a combination of $4 \%$ PAF and $0.1 \%$ glutaraldehyde. However, we found that the intensity of NADPH-d staining was lower when $0.1 \%$ glutaraldehyde was contained in the fixative rather than $0.05 \%$ glutaraldehyde. On the other hand, fixative containing $0.05 \%$ glutaraldehyde produced an intensity of NADPH-d staining comparable to that observed in tissues fixed only with $4 \%$ paraformaldehyde while retaining a level of antigenicity adequate for good GABA and GLU immunolabeling.

\section{Distribution of NADPH-d activity in the IC}

All profiles positive for NADPH-d were characterized by the dark blue-violet formazan reaction product contained in their cytoplasm. Intensely stained neurons, fibers, and puncta were distributed mainly in the cortical subdivisions of the IC (Fig. 1-a). Throughout the dorsal and external cortices (DCIC and ECIC, respectively), small $\left(<100 \mu \mathrm{m}^{2}\right)$, labeled neurons, primarily fusiform or oval in shape, were distributed from layer 1 to layer 3. Especially in transverse sections, many of the layer-1 neurons were oriented parallel to the cortical surface.

Medium-sized (100-150 $\left.\mu^{2}\right)$, NADPH-d positive neurons predominated in the deeper layers, and they possessed fusiform, polygonal or triangular somata with multipolar dendritic arbors, morphological features similar to those of stellate cells (Fig. 1-b, -c). It was apparent from the neutral red counterstaining that the labeled neurons composed only a fraction of the total population of "stellate" neurons. The largest $\left(>200 \mu \mathrm{m}^{2}\right)$ stellate cells, located in the deeper layers of the DCIC and ECIC, were unlabeled (Fig. 1-b, -c).

A uniformly dense staining pattern was evident in the neuropil of the DCIC (Fig. 1-b). In comparison, the labeling of fibers and puncta in the ECIC was discontinuous, particularly in the deeper layers. The distribution of NADPH-d-stained cells, fibers and boutons overlapped one another and appeared to be patchy in comparison to the DCIC (Fig. 1-c).

NADPH-d staining was sparse in the central nucleus of the IC (CIC). A small number of weakly stained, medium-sized, bipolar and multipolar neurons were scattered mostly in the dorsomedial half of the CIC (Fig. 1-a). The labeled bipolar cells did not exhibit preferential alignment along any particular axis.

\section{Combined Staining of NADPH-d Histochemistry with GABA Immunocytochemistry}

Neurons intensely immunopositive for GABA were scattered throughout the IC. These neurons varied considerably in size. The pattern of distribution was similar to that observed in F344 rats by Caspary et al. ${ }^{5)}$ using the same antibody. All GABA-immunolabeled neurons possessed a homogeneous staining profile in which both the nucleus and perikaryon were filled with brown DAB labeling. Immunonegative neurons possessed no staining in either compartment. Thus, in the double-labeled sections, cells exhibiting co-labeling for GABA and NADPH-d were readily distinguishable by the blue-black NADPH-d reaction product contained in the perikarya and the brown GABA immunostaining located in the nuclei.

The majority of GABA/NADPH-d-colabeled neurons were small cells $(<100 \mu \mathrm{m})$ (Table 1) possessing round or oval somata, usually with a bipolar dendritic organization. They were distributed mainly in layer 2 of the DCIC and ECIC (Fig. 2-a, -b). There were no NADPH-d positive cells in the central nucleus of the IC (CIC) that co-labeled for GABA. Cell counts from midsagittal IC sections obtained from five F344 rats (Fig. 3) revealed

Table 1. Cell size distribution of neurons double-labeled with NADPH $-d$ and GABA.

All NADPH-d positive neurons (NADPH-d+/0) are composed of cells ranging in size less than $200 \mu \mathrm{m}^{2}$, especially more than $60 \%$ are smaller than $100 \mu^{2}$. NADPH-d/GABA colabeled neurons (NADPH-d+/GABA+) measure no more than 130 $\mu^{2}$ and the majority of them (over $80 \%$ ) are found less than $100 \mu \mathrm{m}^{2}$. There are also some occasional medium cells in layer 3 containing both GABA and NADPH-d, however the most of NADPH-d/GABAergic neurons seemed to belong to the small cells group (somatic area $<100 \mu \mathrm{m}^{2}$ ).

\begin{tabular}{lccccc}
\hline & \multicolumn{5}{c}{ Somatic area $\left(\mu \mathrm{m}^{2}\right)$} \\
\cline { 2 - 6 } & $<100$ & $100-129$ & $130-149$ & $150-199$ & $\geq 200$ \\
\hline NADPH+/0 & 685 & 315 & 52 & 17 & 1 \\
$(5$ animals, $n=1070)$ & $(64.0 \%)$ & $(29.4 \%)$ & $(4.9 \%)$ & $(1.6 \%)$ & $(0.1 \%)$ \\
NADPH+/GABA+ & 96 & 23 & 0 & 0 & 0 \\
$(5$ animals, $n=119)$ & $(80.7 \%)$ & $(19.3 \%)$ & & & \\
\hline
\end{tabular}




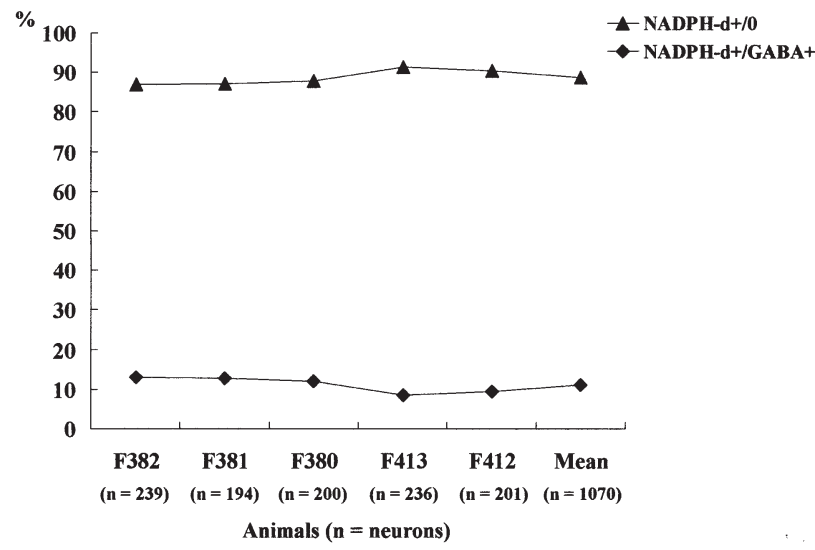

Fig. 3. A diagram of double labeling for NADPH-d and GABA.

Showing the relative percentages of NADPH-d/GABA colabeled neurons (NADPH-d $+/ \mathrm{GABA}+$ ) and NADPH-d single-labeled neurons (NADPH-d+/0) in the total population of NADPH-d containing neurons (set to $100 \%$ ). Cell count is carried out for each section through the mediolateral middle of the IC of 5 animals and reveals that a small population; approximate $11.2 \%$ of NADPH-d containing neurons are also GABA immunoreactive.

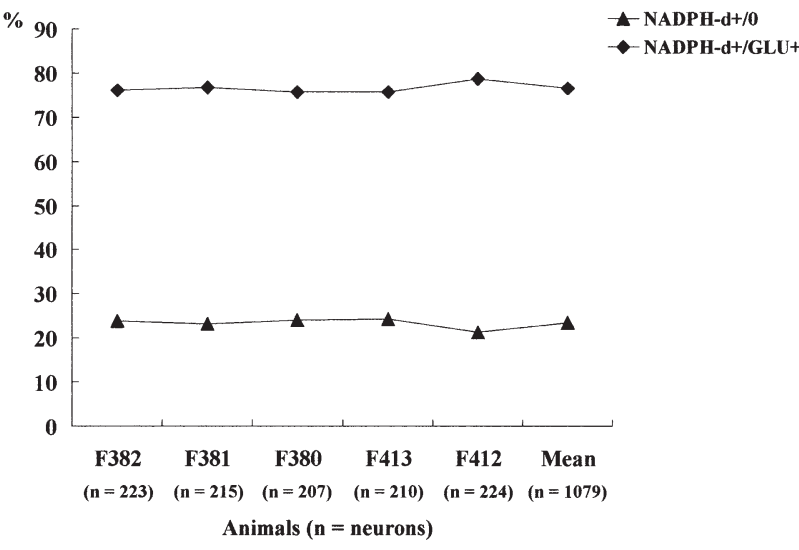

Fig. 4. A diagram of double labeling for NADPH-d and GLU.

Showing the relative percentages of NADPH-d/GLU colabeled neurons (NADPH-d+/GLU+) and NADPH-d single-labeled neurons (NADPH-d $+/ 0$ ) in the total population of NADPH-d positive neurons (set to $100 \%$ ). Cell count is carried out for each section through the mediolateral middle of the IC of 5 animals and reveals that the greater part, approximate $76.6 \%$ of NADPH-d containing neurons are also GLU immunoreactive.
Table 2. Cell size distribution of neurons double-labeled with NADPH $-d$ and GLU.

Similar to the somatic distribution of NADPH-d positive neurons (NADPH- $\mathrm{d}+/ 0$ ), a little more than $60 \%$ of NADPH- $\mathrm{d} /$ GLU colabeled neurons (NADPH-d+/GLU+) measure less than $100 \mu \mathrm{m}^{2}$, and about $30 \%$ measure from 100 to $129 \mu \mathrm{m}^{2}$. There are also some larger cells ranging from 130 to 200 $\mu \mathrm{m}^{2}$, however no one is found greater than $200 \mu \mathrm{m}^{2}$, in somata.

\begin{tabular}{lccccc}
\hline & \multicolumn{5}{c}{ Somatic area $\left(\mu \mathrm{m}^{2}\right)$} \\
\cline { 2 - 6 } & $<100$ & $100-129$ & $130-149$ & $150-199$ & $\geq 200$ \\
\hline NADPH+/0 & 676 & 320 & 61 & 22 & 0 \\
$(5$ animals, $\mathrm{n}=1079)$ & $(62.7 \%)$ & $(29.7 \%)$ & $(5.7 \%)$ & $(2.0 \%)$ & 0 \\
NADPH+/GLU+ & 506 & 257 & 49 & 15 & 0 \\
$(5$ animals, $\mathrm{n}=827)$ & $(61.2 \%)$ & $(31.1 \%)$ & $(5.9 \%)$ & $(1.8 \%)$ & 0 \\
\hline
\end{tabular}

that $11.2 \% \pm 0.92 \%$ (S.E.M) of the total population of NADPH-d containing neurons also were GABA immunoreactive.

Combined Staining of NADPH-d Histochemistry with GLU Immunocytochemistry

Neurons moderately-to-intensely immunoreactive for GLU were distributed extensively throughout the IC. They existed in a wide variety of shapes and sizes, but mostly they were small-to-medium sized, and round or polygonal in shape. The density of glutamate-immunopositive neurons was greatest in the DCIC, followed by the ECIC and CIC in decreasing order. These findings agree with previous biochemical and immunohistochemical showing that the cortical regions of the IC contained higher transmitter levels of GLU ${ }^{2,19)}$.

The immunostaining properties of GLU were similar to those observed with GABA. The cytoplasm and nuclei of GLU immunoreactive neurons were uniformly stained. Again, neurons coexpressing NADPH-d and GLU were detectable by virtue of a dark blue cytoplasmic precipitate and an immunostained nucleus.

Most of the NADPH-d positive neurons colabeled for GLU (76.6\% $0.53 \%$; Fig. 4). This was most evident in the cortical regions of the IC where, as previously mentioned, the majority of NADPH-d positive neurons are located (Fig. 5-a, -b). From a cytoarchitectonic standpoint, the NADPH/GLU colabeled neurons possessed a wider range of somal sizes (small to large (Table 2) and shapes (fusiform to polygonal) than did the NADPH-d/ GABAergic neurons, and included the population of neurons that could be characterized as stellate cells.

We were unable to detect GABA or GLU in a small percentage of the NADPH-d positive neurons, and we did not observed NADPH neurons that were intensely colabeled with both transmitters (Fig. 6-A, -a, Fig. 6-B, -b). Thus, NOS-containing IC neurons can be subdivided into three groups based on transmitter specificity, with the largest proportion $(\sim 77 \%)$ containing GLU, but not GABA. The remainder is divided into roughly equal portions of neurons immunopositive for GABA but not glutamate, and those containing neither (Fig. 7). 


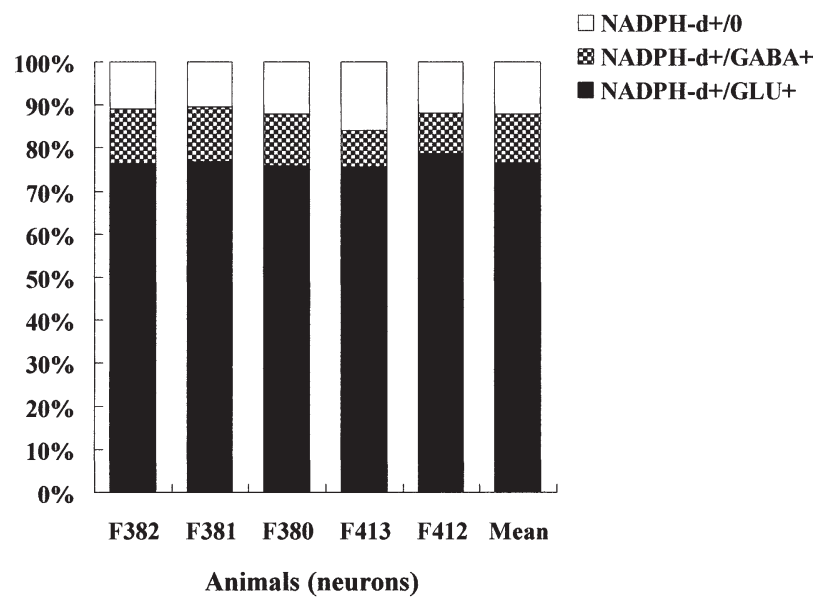

Fig. 7. A diagram showing the relative proportions of neurons colabeled for NADPH-d and GABA (NADPH- + +/GABA + ), and for NADPH-d and GLU (NADPH-d+/GLU+).

For each bar, the percentages of both colabeled groups of NADPH-d+/GABA+ and NADPH-d+/GLU+ were referred to previous Fig. 3 and Fig. 4 respectively, and the remainder of percentage point was counted for NADPHD-d single-positive group (NADPH-d+/0). Because that no coexistence of GABA and CLU could be found in the same cells according to the mirror-images technique, the mean corresponding ratio from 5 individual animals between these three subpopulations of NOergic neurons is NADPH-d+/GLU+, 8 (76.6\%); NADPH-d/GABA+, $1(11.2 \%)$; and NADPH-d+/0, $1(12.2 \%)$.

\section{Discussion}

Recently several investigators have described the distribution of NOergic neurons in relation to aminergic neurons in different regions of the central nervous system, including the zona inserta ${ }^{17}$, prefrontal $\operatorname{cortex}^{13)}$, medulla oblongata ${ }^{21)}$, hippocampus ${ }^{37}$, , cuneate nucleus ${ }^{35}$, olfactory bulb ${ }^{1)}$, lateral geniculate nucleus ${ }^{14)}$, spinal cord $^{31)}$, and cerebral cortex ${ }^{36}$. Nevertheless, little is known about the relationship between NO and neuroactive amino acids in the auditory nuclei. In the present study, the combined staining of NADPH-d histochemistry with GABA or GLU immunohistochemistry was utilized to investigate a potential co-existence of $\mathrm{NO}$ with GABA and GLU in the IC of rat.

Formaldehyde fixation has been established as a reliable preservation technique for the histochemical detection of NADPH-d. Indeed, a previous study by the authors demonstrated complete overlap between NADPH-d labeling and immunoreactivity for brain NOS (bNOS) in sections of IC perfusion fixed with buffered $4 \%$ paraformaldehyde ${ }^{39)}$. Nevertheless, the present study has demonstrated that fixatives containing small concentrations $(0.05 \%)$ of glutaraldehyde in addition to paraformaldehyde permit the immunodetection of amino acid conjugates without seriously compromising NADPH-d staining. However, with progressively higher concentrations of glutaraldehyde, we found that the staining for NADPH-d became increasingly less intense such that increasing numbers of false negatives would certainly result. On the other hand, the low levels of glutaraldehyde in our fixatives produced patterns of GABA and GLU immunolabeling in the IC comparable to those previous reports $^{2,5,26)}$.

Relationship between NADPH-d staining and cell types in the IC

The greatest number of intensely NADPH-d-stained neurons were located in layers 2 and 3 of the DCIC and ECIC. Based on their cytoarchitectural features, these neurons appear to be stellate cells that have been identified as the predominate neurons in the IC cortices ${ }^{11,24}$. The responses of stellate cells could reflect the synthesis of information from several synaptic domains suggesting that this type of cells may contribute to the intrinsic axonal plexus of the IC and also play a part in efferent projections ${ }^{25)}$. As like the cortex of IC has been established to be a unique auditory relay station in which, much different from CIC, both of the ascending and descending inputs from the lower auditory brainstem and the telencephalon terminated, there was a suggestion of stellate cells playing an important role in connections of ascending and descending auditory pathways. And also ECIC was characterized as an acousticomotor nucleus, extensively interconnecting with the superior colliculus, cerebellum and somatosensory and vocalization systems $\mathrm{s}^{6,16,25,32)}$. As determined by our previous quantitative study ${ }^{39}$, NADPH-d-stained neurons account for approximately $35 \%$ of the total neuronal population in the IC cortices. Thus, we believe that a prominent subpopulation of stellate neurons in the central and cortical IC is NOergic. It is presumptive that the NOergic neurons in the IC cortices, mostly corresponding to stellate cells, may participate in integrating the ascending and descending auditory pathways as well as possibly contribute to multiple connections between the auditory system and other somatosensory or motor systems, however, the precise neurophysiologic role of these neurons, along with the significance of NO in this capacity, remains to be determined.

The overlapping, patchy distribution of NADPH-dlabeled cells, fibers and puncta characterized the ECIC from the other IC subdivisions. Interestingly, the discontinuous distribution of the labeled fibers and boutons is reminiscent of that observed for the somatosensory projections to the same region ${ }^{11)}$. However, the coincident appearance of NADPH-d labeled cells along with the fibers and puncta in discrete areas of the ECIC suggest that NOergic neurons function here as local circuit neurons. 


\section{Co-localization of NADPH-d and amino acid neurotrans-} mitters in the same cells

Three types of NOS-containing neurons were identified in the IC based on whether or not they co-contained GABA or GLU. Approximately $77 \%$ of the NOergic neurons colabeled for GLU and $\sim 11 \%$ were immunopositive for GABA, while the remainder appeared to contain neither transmitter (Fig. 7).

It is now evident that GABA is present at high concentrations in the IC, supporting a possible role of GABA as an inhibitory transmitter in this nucleus ${ }^{2,12)}$. Immunohistochemical studies have shown that GABAergic neurons are found scattered throughout the IC, and they appear to be a subpopulation of small- to medium-sized stellate neurons ${ }^{27)}$ that are thought to be involved in local circuit activity ${ }^{28,29)}$. Perhaps a small percentage of these neurons are also NOergic, capable of modifying intrinsic inhibitory circuits which shape the processing of acoustic information in the IC cortices ${ }^{5}$.

As with GABA, high concentrations of GLU are also found in the $\mathrm{IC}^{2,10,26)}$ that has proposed a role of GLU as a candidate for the afferent excitatory transmitter in the IC where excitatory amino acid application has been examined to induced a reduction of the acoustic threshold in several IC neurons. On the other hand, several studies have suggested that NO may co-exist with GLU in neuronal groups in the cerebellum ${ }^{3)}$, medulla ${ }^{21)}$ and thalamus $^{17)}$ suggesting a complex interaction between these two messenger molecules and implying another different excitatory mode of $\mathrm{NO}$ transmission from what was inhibitorily modulated by GABA. However, given their smaller size and morphologic similarity to stellate cells NO/GLUergic cells may function as interneurons in the IC. With antagonizing to the inhibitory effects of NO/GABAergic neurons, a GLU-mediated excitatory NO transmission is presumed to be involved in the networking of auditory communication in the cortex of IC.

In Addition, it is also interesting to note that the CA1 pyramidal neurons in the hippocampus, supposed to be the source of long-term potentiation (LTP) has been identified as $\mathrm{NO} / \mathrm{GLUergic}^{7,26)}$, while an exclusive local circuit population also proposed to be related to the LTP induction were found $\mathrm{NO} / \mathrm{GABAergic}{ }^{36)}$. In the cerebellum, two types of inhibitory interneurons, i.e. the stellate and basket cells, presumably concerned in regulating the effect of long-term depression (LTD) were also found $\mathrm{NO} / \mathrm{GABAergic}^{36)}$. At present, the knowledge concerning the synaptic plasticity such as LTP and LTD in auditory pathways is still deficient, however, $\mathrm{NO} / \mathrm{GABAergic}$ and $\mathrm{NO}$ /GLUergic interneurons respectively provide a possibility that an inhibitory GABA- or an excitatory GLUmediated form of synaptic plasticity may be associated with the progresses of acoustic learning or recognition memory in the IC, whereas different effects are probably produced by NOergic simple neurons which contain neither GABA nor GLU.

\section{Reference}

1) Alonso JR, Arévalo R, García-Ojeda E, Porteros A and Briñón JG. NADPH-diaphorase active and calbindin D-28k-immunoreactive neurons and fibers in the olfactory bulb of the hedgehog (Erinaceus europaeus ). J Comp Neurol 1995; 351:307-327.

2) Adams JC and Wenthold RJ. Distribution of putative amino acid transmitters, choline acetyltransferase and glutamate decarbozylase in the inferior colliculus. Neuroscience 1979; 4:19471951.

3) Bredt DS, Glatt, CE, Hwang PM, Fotuhi MT, Dawson M and Snyder SH. Nitric oxide synthase protein and mRNA are discretely localized in neuronal populations of the mammalian CNS together with NADPH diaphorase. Neuron 1991; 7:615-624.

4) Bredt DS, Hwang PM and Snyder SH. Localization of nitric oxide synthase indicating a neural role for nitric oxide. Nature 1990; 347:768-770.

5) Caspary DM, Raza A, Lawhorn Armour BA, Pippin J and Arneric SP. Immunocytochemical and neurochemical evidence for agerelated loss of GABA in the inferior colliculus: implication for neural presbycusis. J Neurosci 1990; 10:2363-2372.

6) Coleman J and Clerici WJ. Sources of projections to subdivisions of the inferior colliculus in the rat. J Comp Neurol 1987; 262:215 -226 .

7) Dinerman JL, Dawson TM, Schell MJ and Snowman A. Endothelial nitric oxide synthase localized to hippocampal pyramidal cells: Implications for synaptic plsasticity. Proc Natl Acad Sci USA 1994; 91:4214-4218.

8) Druga R and Syka J. NADPH-diaphorase activity in the central auditory structures of the rat. Neuroreport 1993; 4:999-1002.

9) Dawson TM, Bredt DS, Fotuhi M, Hwang PM and Snyder SH. Nitric oxide synthase and neuronal NADPH diaphorase are identical in brain and peripheral tissues. Proe Natl Acad Sci USA 1991; 88:7797-7801.

10) Faingold CL, Gehlbach G and Caspary DM. Functonal pharmacology of inferior colliculus neurons. In Altschuler RA, Bobbin RP, Clopton BM, Hoffman DW, eds, Neurobiology of hearing: The central auditory system. Raven Press, New York, 1991; 223 251.

11) Faye-Lund $\mathrm{H}$ and Osen KK. Anatomy of the inferior colliculus in. Anat Embryol 1985; 171:1-20.

12) Fisher SK and Davies WE. GABA and its related enzymes in the lower auditory system of the guinea pig. J Neurochem 1976; 27:1145-1155.

13) Gabbott PLA, Dickie BG, Roy Vaid R, Headlam AJ and Bacon SJ. Local-circuit neurones in the medial prefrontal cortex (areas 25, 32 and 24b) in the rat: Morphologiy and quantitative distribution. J Comp Neurol 1997; 377:465-499.

14) Gabbott PLA and Bacon SJ. Two types of interneuron in the dorsal lateral geniculate nucleus of the rat: A combined NADPH diaphorase histochemical and GABA immunocytochemical study. J Comp Neurol 1994-a; 350:281-301.

15) Garthwaite J, Charles SL and Chess-Williams R. Endotheliumderiived relaxing factor release on activation of NMDA receptors suggests role as intercellular messenger in the brain. Nature 1988; 336:385-388.

16) Huffman RF and Henson Jr OW. The descending auditory pathway and acousticomotor systems: connections with the inferior colliculus. Brain Res Revi 1990; 15:295-323.

17) Kolmac C and Mitrofanis J. Distribution of various neurochemicals within the zona incerta: an immunocytochemical and histochemical study. Anat Embryol 1999; 199:265-280.

18) Kimura M, Wu M, Inafuku S and Ishigami H. Age-related changes in the immunohistochemical localization of inducible nitric oxide synthase (iNOS) and nuclear factor-kappa B (NF- $\kappa \mathrm{B})$ in the mouse cochlea. Acta Histochem Cytochem 1998; 31:493-500.

19) Kaneko T, Itoh K, Shigemoto R and Mizuno N. Glutaminase-like 
immunoreactivety in the lower brainstem and cerebellum of the adult rat. Neuroscience 1989; 32:79-98.

20) McGinn MD and Cox RG. Nitric oxide synthetase staining in the cochlear nucleus and superior olive of the Mongolian gerbil. Society Neurosci 1995; 21:402.

21) Maqbool A, Batten TF and McWilliam PN. Co-localization of neurotransmitter immunoreactivities in putative nitric oxide synthesizing neurones of the cat brain stem. J Chem Neuroanatomy 1995; 8:191-206.

22) Moore JK and Moore RY. Glutamic acid decarboxylase-like immunoreactivity in brainstem auditory nuclei of the rat. J Comp Neurol 1987; 260:157-174.

23) Oliver DL, Winer JA, Beckius GE and Saint Marie RL. Morphology of GABAergic neurons in the inferior colliculus of the cat. J Comp Neurol 1994; 340:27-42.

24) Oliver DL and Hueta RL. Inferior and superior colliculus. In Wester DB, Popper AN, Fay RR, eds, The mammalian auditory pathway: Neuroanatomy. Springer-Verlag, New York, 1992; 168221.

25) Oliver DL and Shneiderman A. The anatomy of the inferior colliculus: A cellular basis for integration of monaural and binaural information. In Altschuler RA et al eds, Neurobiology of hearing: The central auditory system. Raven Press, New York, 1991; 195222.

26) Ottersen OP and Storm-Mathisen J. Neurons containing or accumulating transmitter amino acids. In Björklund A, Hökfelt T, Kuhar MJ, eds, Handbook of chemical neroanatomy, vol. 3. Classical transmitters and transmitter receptors in the CNS, part II. Elsevier, Amsterdam, 1984; 141-246.

27) Roberts RC and Ribak CE. An electron microscopic study of GABAergic neurons and terminals in the central nucleus of the inferior colliculus of the rat. J Neurocyto 1987; 16:333-345.

28) Rockel AJ and Jones EG. The neuronal organization of the inferior colliculus of the adult cat. I. The central nucleus. J Comp Neurol 1973-a; 147:11-60.

29) Rockel AJ and Jones EG. Observations on the fine structure of the central nucleus of the inferior colliculus of the cat. J Comp Neurol 1973-b; 147:61-92.

30) Sánchez F, Alonso JR, Arévalo R, Blanco E, Aijón J and Vázquez
R. Coexistense of NADPH-diaphorase with vasopress in and oxytocin in the hypothalamic magnocellular neurosecretory nuclei of the rat. Cell Tissue Res 1994; 276:31-34.

31) Spike RC, Todd AJ and Johnston HM. Coexistence of NADPH diaphorase with GABA, glycine, and acetylcholine in rat spinal cord. J Comp Neurol 1993; 335:320-333.

32) Saldaña $E$ and Merchán MA. Intrinsic and Commissural connections of the rat inferior colliculus. J Comp Neurol 1992; 319:417 $-437$.

33) Scherer-Singler U, Vincent SR, Kimura $\mathrm{H}$ and McGeer EG. Demonstration of uniquepopulation of neurons with NADPHdiaphrase histochemistry. J Neuroscience Methods 1983; 9:229234.

34) Sokoloff L, Reivich M, Kennedy C. The $\left({ }^{14} \mathrm{C}\right)$ deoxyglucose method for the measurement of local cerebral glucose utilization: theory, procedure, and normal values in the conscious and anaesthetized albino rat. J Neurochem 1977; 28:897-916.

35) Valtschanff JG, Weinberg RJ, Rustioni A and Schmidt HHHW. Colocalization of neuronal nitric oxide synthase with GABA in rat cuneate nucleus. J Neurocytol 1995; 24:237-245.

36) Valtschanoff J G, Weinberg RJ, Kharazia VN, Schmidt HHHW, Nakane $\mathrm{M}$ and Rustioni A. Neurons in rat cerebral cortex that synthesize nitric oxide: NADPH diaphrase histochemistry, NOS immunocytochemistry, and colocalization with GABA. Neurosci Lett 1993-a; 157:157-161.

37) Valtschanoff JG, Weinberg RJ, Kharazia VN, Nakane M and Schmidt HHHW. Neurons in hippocampus that synthesize nitric oxide. J Comp Neurol 1993-b; 33:111-121.

38) Vincent SR and Kimura H. Histochemical mapping of mitric oxide synthase in the rat brain. Neurosciece 1992; 46:755-784.

39) Wu M, Nakayama M, Ishigami H, Helfert RH and Konrad HR. The distribution of NOergic neurons in the auditory pathway of the rat. Otol Jpn 1999; 9:542-550 (in Japanese).

40) Wu M, Kimura M, Inafuku S and Ishigami $\mathrm{H}$. Effect of aging on the expression of iNOS and cell death in the mouse cochlear spiral ganglion. Okajima Folia Anat Jpn 1997; 74:155-166.

41) Wenthold RJ, Zemple JM, Parakkal MH, Reeks KA and Altschuler RA. Immunocytochemical localization of GABA in the cochlear nucleus of the guinea pig. Brain Res 1986; 380:7-18. 

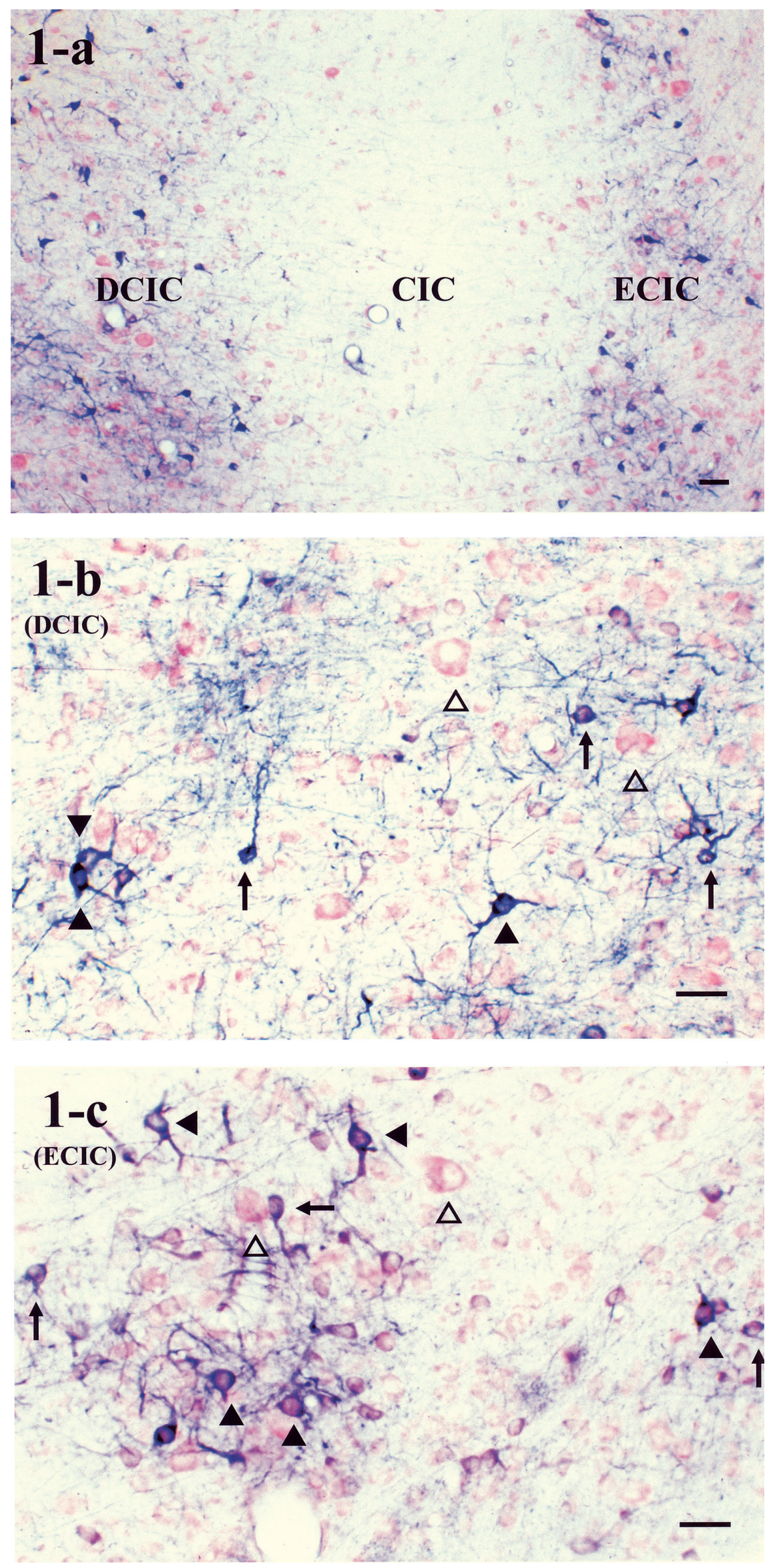

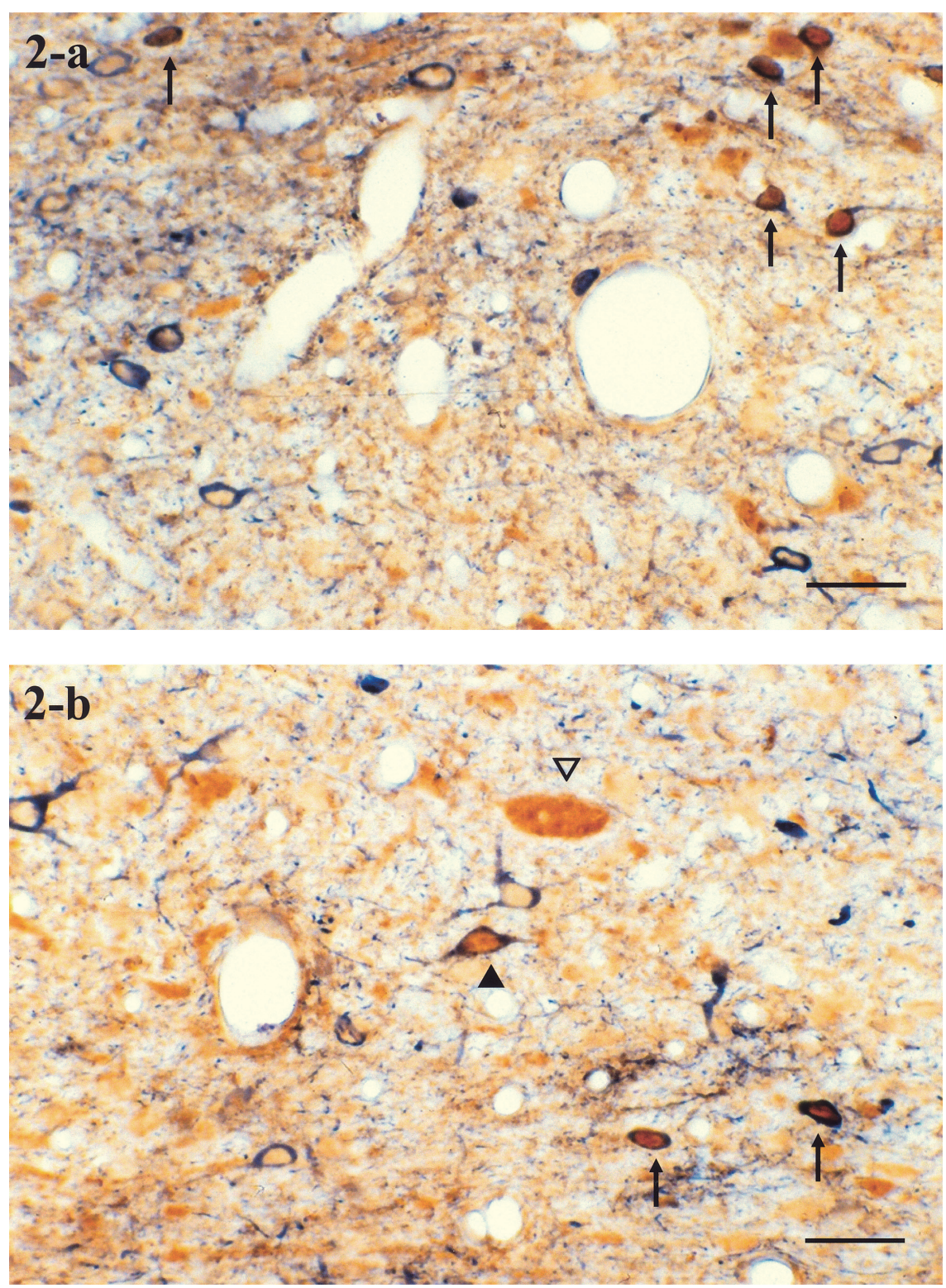

Fig. 2. Combined staining of NADPH-d histochemistry with GABA immunohistochemistry in the IC.

A small number of double-labeled NADPH-d/GABAergic neurons, mostly possessing small round or oval soma (arrows) are found in the layer 2 of DCIC (2-a) and ECIC (2-b). In the layer 3 of ECIC, some occasional medium cells (arrowheads) also exhibit colocalization of NADPH-d and GABA; however the giant neurons (open arrowhead) are labeled with GABA immunohistochemistry but lack NADPH-d reactivity (2-b). Scale bar $=50 \mathrm{um}$.

Fig. 1. NADPH-d histochemistry in the IC.

1-a: The NADPH-d positive neuropil and neurons, which are characterized by the blue-violet formazan formation throughout their cytoplasm with a Golgi-like aspect, are predominantly found in the dorsal cortex (DCIC) and the external cortex (ECIC). Only a few NADPH-d labeled neurons thinly disperse in the central nucleus of the IC (CIC), however a number of labeled fibers are observed in this area bridging the DCIC and ECIC. 1-b: A part of deeper layer of DCIC enlarged. NADPH-d positive neurons with small (arrows) to medium-sized (arrowheads) somata and irregular dendritic orientations, and neuropil are rich in the DCIC. The largest stellate cells (open arrowheads) are unlabeled. 1-c: In comparison to the DCIC (1-b), cells (the small are indicated by arrows and the medium-sized by arrowheads), fibers and boutons are less marked in the ECIC and had a tendency to be arranged in clusters especially in layer 2. The largest stellate cells (open arrowheads) are unlabeled. Scale bar $=50$ um all photomicrographs. 

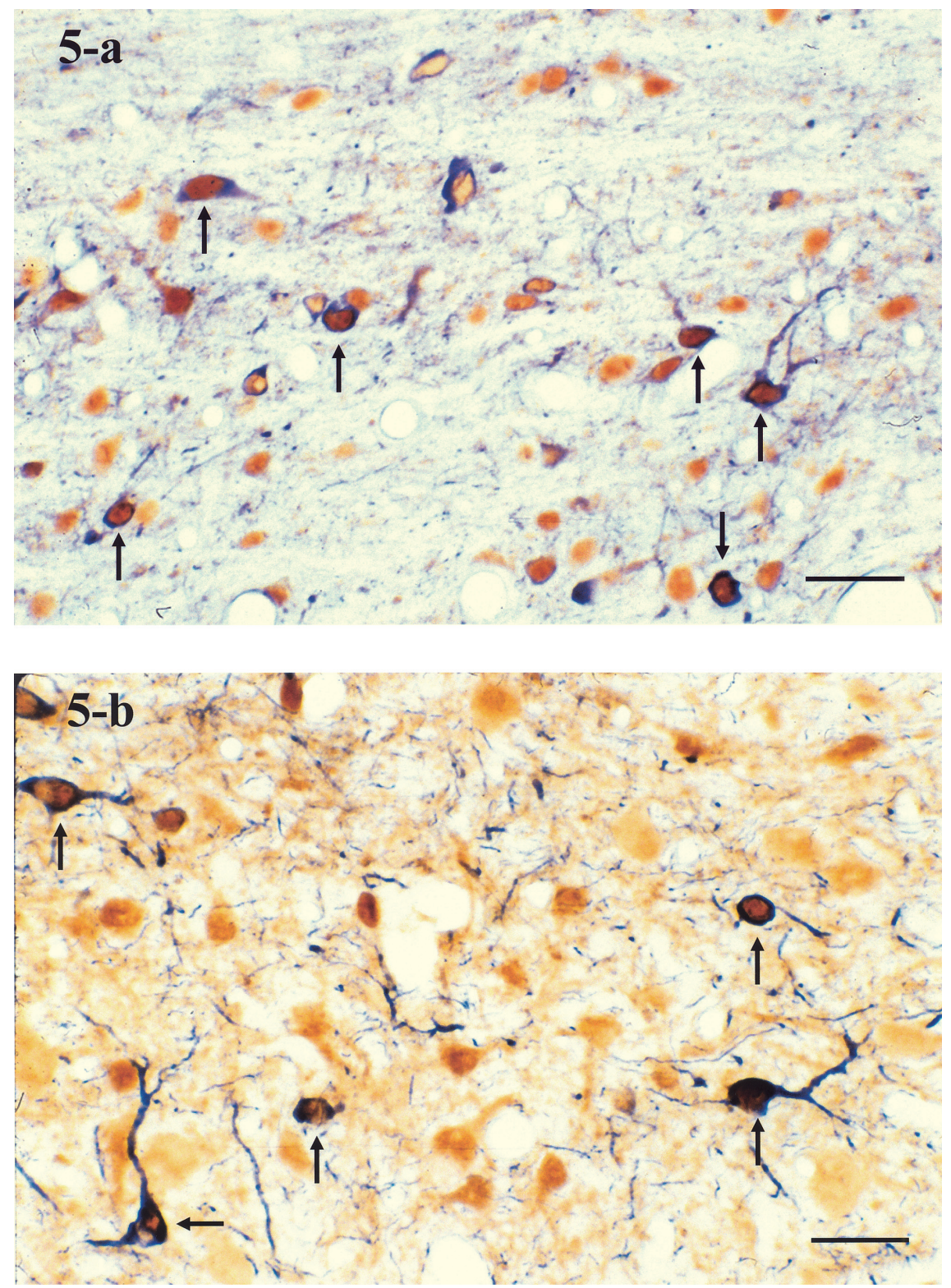

Fig. 5. Combined staining of NADPH-d histochemistry with GLU immunohistochemistry in the IC.

A majority of NADPH-d/GLUergic neurons showing NADPH-d reactive and intensive or moderate (arrows) GLU-immunoreactivity are detected in the DCIC (4-a) and ECIC (4-b). From the cytoarchitectonic perspective, these NADPH-d/GLUergic neurons possess a wider range of somal sizes (small to large) and shapes (fusiform to polygonal) than did the NADPH-d/GABAergic neurons, and include the population of neurons that could be characterized as stellate cells. Scale bar $=50 \mathrm{um}$. 


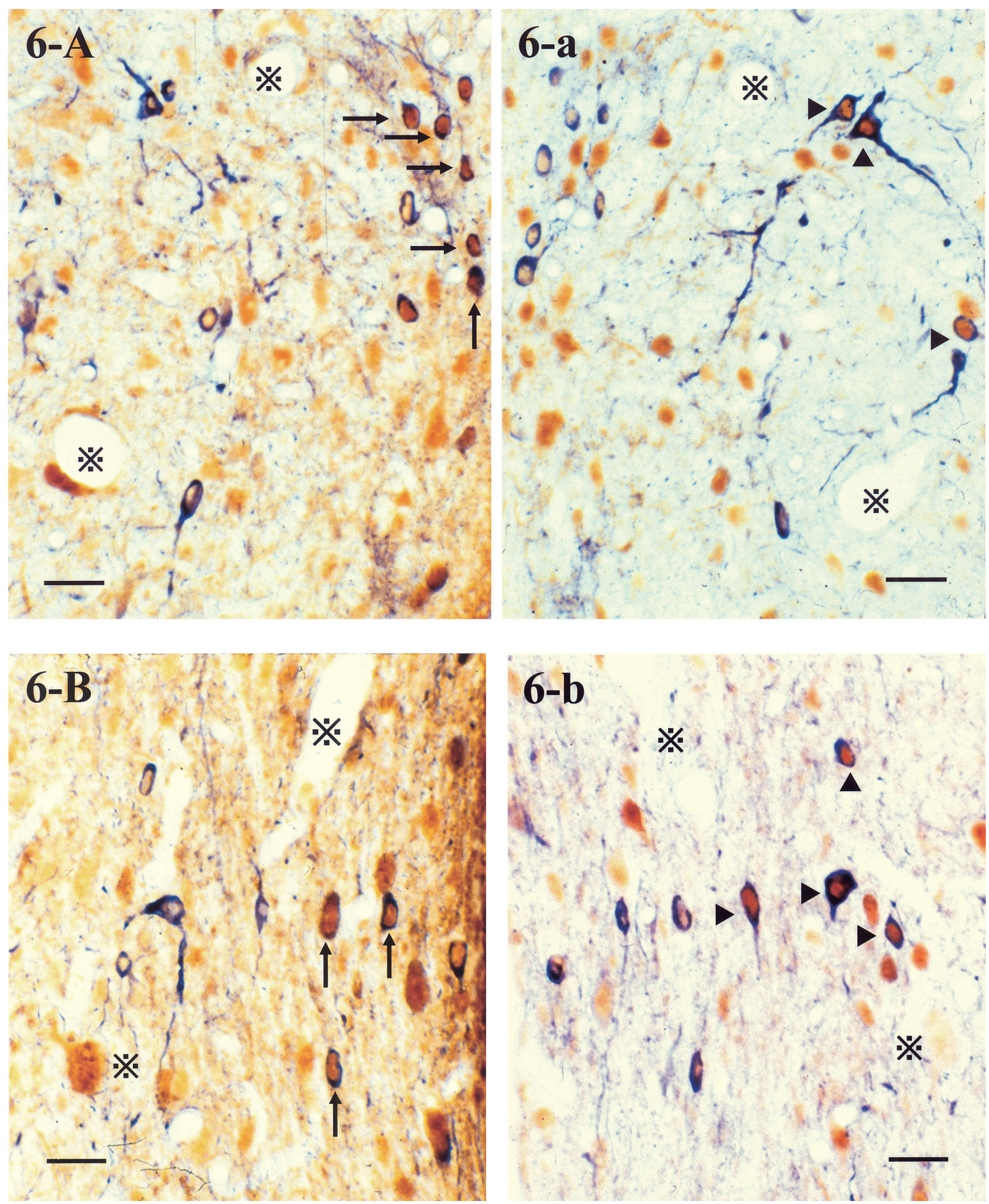

Fig. 6. The pair adjacent sections consisted of one section colabeled for NADPH-d and GABA, and the other one for NADPH-d and GLU. Adjacent surfaces of the pairs of section 6-A and 6-a, and the pairs of section 6-B and 6-b are examined as mirror-images. 6-A and 6-B are colabeled for NADPH-d and GABA, and 6-a and 6-b are colabeled for NADPH-d and GLU respectively.

Note that all of NO/GABAergic neurons (arrows) seen in 6-A and 6-B do not show any GLU immunoreactivity in 6-a and 6-b. In contrast, the NO/GLUergic neurons (arrowheads) seen in 6-a and 6-b are all GABA immunonegative. Asterisks indicate profiles of blood vessels as landmarks. Scale bar $=50$ um for all photomicrographs. 